\title{
Bleeding recognition technique in wireless capsule endoscopy images using fuzzy logic and principal component analysis
}

\author{
A. Al Mamun', P. P. Em², T. Ghosh ${ }^{3}$, M. M. Hossain ${ }^{4}$, M. G. Hasan ${ }^{5}$, M. G. Sadeque \\ ${ }^{1,2}$ Faculty of Engineering and Technology, Multimedia University, Malaysia \\ ${ }^{3}$ Department of Electrical and Computer Engineering, University of Alabama, United States \\ ${ }^{4,5,6}$ Department of EEE, Pabna University of Science and Technology, Bangladesh
}

\begin{tabular}{l} 
Article Info \\
\hline Article history: \\
Received Aug 27, 2020 \\
Revised Sep 25, 2020 \\
Accepted Oct 28, 2020 \\
\hline
\end{tabular}

\section{Keywords:}

Bleeding detection

Fuzzy logic

Gastrointestinal tract

Principal component analysis

QSVM

Wireless capsule endoscopy

\begin{abstract}
Wireless capsule endoscopy is the most innovative technology to perceive the entire gastrointestinal (GI) tract in recent times. It can diagnose inner diseases like bleeding, ulcer, tumor, Crohn's disease, and polyps in a discretion way. It creates immense pressure and onus for clinicians to perceive a huge number of image frames, which is time-consuming and makes human oversight errors. Therefore a computer-automated system has been introduced for bleeding detection. A unique fuzzy logic technique is proposed to extract the specified bleeding and non-bleeding information from the image data. A particular quadratic support vector machine (QSVM) classifier is employed to classify the obtained statistical features from the bleeding and non-bleeding images incorporating principal component analysis (PCA). After extensive experiments on clinical data, 98\% sensitivity, $98.4 \%$ accuracy, $98 \%$ specificity, $93 \%$ precision, $95.4 \% \mathrm{~F} 1$-score, and $99 \%$ negative predicted value have been achieved, which outperforms some of the states of art methods in this regard. It is optimistic that the proposed methodology would significantly contribute to bleeding detection techniques and diminish the additional onus of the physicians.
\end{abstract}

This is an open access article under the CC BY-SA license.

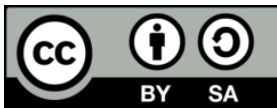

Corresponding Author:

Abdullah Al Mamun

Faculty of Engineering and Technology

Multimedia University

Ayer Keroh, Melaka-7540, Malaysia

Email: mamun130203@gmail.com

\section{INTRODUCTION}

The diseases of the Gastrointestinal tract in the large intestine, small intestine, and stomach have turned into the most epidemic problem in modern life since it involves different diseases like bleeding, ulcer, tumor, and cancer [1]. Due to different types of difficulties and restrictions, conventional endoscopes are unable to extract the information from the inner portion of the gastrointestinal tract [2]. A majority number of people from all over the world are undergoing the severe effect of GI tract diseases. Around 2.1 people per thousands have died for IBD (inflammatory bowel disease), and 5\% of the western population have been suffering from the $\mathrm{c}$ disease [3]. In another observation, around 1.6 million people from American have been affected and died for IBD, and approximately 10 million babies have been suffered from Crohn's disease [4]. So, efficient solutions need to be introduced to cure gastrointestinal tract problems as soon as possible. After the development and revolution of optic endoscopy, the diagnosis and therapy processes have been received the touch of growth in the gastrointestinal tract diseases. But it is a matter of regret that only a small portion of the proximal duodenum and bowel can be examined. In 2000, a revolution was created in the field of endoscopy by introducing capsule endoscopy from the given imaging company. WCE has been applied for 
the real-time application in 2001. The capsule endoscopy can visualize the small intestine and introduce modern technology to snap from the gastrointestinal tract by a swallowed wireless capsule. The FDA has certified capsule endoscopy for safety and acceptability [5].

Wireless capsule endoscopy consists of an image sensor, transmitter, battery, light, and color camera. It requires almost eight hours and snaps around $57 \mathrm{~K}$ images to complete the travel throughout the whole gastrointestinal tract. The snapped images are transmitted to a particular computer that is operated by a certain radio-frequency. The physicians require checking out the sent images to find out the disease indications, and it becomes too much difficult to sort out the precise information of the diseases from the images manually for the sack of irregular distribution of the particular information and human oversight difficulties. Therefore, the researchers are trying to introduce a specific technique to detect the bleeding portion automatically [6].

\section{RELATED WORK}

Bleeding detection is vital for the clinical perspective because many of the GI tract disease recognitions depend on it [2]. Distinct types of computer-aided systems have already been introduced into WCE images to deduce the burden of the physicians for detecting the bleeding. However, these techniques provided unsatisfactory results in terms of sensitivity and specificity, such as suspected blood indicator [7]. Initially, a framework was designed to detect bleeding portion in which the specificity and sensitivity were only $41.8 \%$ and $21.5 \%$. According to [8], Probabilistic neural network is applied to detect the bleeding portion and also improved to recognize bleeding part for achieving more precise results. A superpixel technique has been applied by Sivakumar et al. [9] with a Naive Bayes classifier to detect the bleeding region accurately. However, the model has been trained only two statistical features and did not validate with the other exiting techniques. The information loss can be reduced up to a significance level by using multiple random training datasets and achieved higher specificity and sensitivity by applying the support vector machine.

Konstantin et al. have introduced a bleeding detection technique utilizing the texture and color features that would provide the complete color information. Nevertheless, the color information technology provides lower performance results compared to the other existing methods [10]. The frequency spectrum of characteristics pattern is based on the normalized gray level co-occurrence matrix and achieved a satisfactory bleeding detection rate [11]. A unique two-fold system is introduced to detect the bleeding portioning in which K-means clustering and SVM classifier has been applied to extract the cluster center and distinguish the bleeding images correspondently [12]. A changeable color domain has been implemented instead of the RGB color model to reduce the computational time. The support vector machine has been applied to the statistical features depending on higher and lower values to classify the bleeding and non-bleeding image efficiently [2]. In [13], the authors extracted different color features from the images by employing the histogram technique, and SVM classifier applied to distinct images. Authors proposed in [14] a compound model named Y.I/Q to extract the information about the chrominance and luminance for acquiring the region of interest of the images and the SVM classifier technique employed for a satisfactory result. In [15], authors formed a pixel intensity ration of R/G (Red/Green) from the RGB color model to achieve different statistical features and K- nearest neighbor classifier used for classification. Authors employed intensity fluctuation of the pixels in the RGB color model with statistical characteristics analysis in [16]. Though these techniques have done tremendous work on bleeding detection from WCE images, these still have the limitation of computational complexity and lower performance results. Color threshold techniques on different color spaces have been applied for detecting different abnormalities. However, it couldn't distinguish the informative portion from the background for all the images [17-22].

In this paper, an exemplary accurate bleeding detection technique is introduced from wireless capsule endoscopy images in the HSV color space. Firstly, the WCE images are received by transforming the video of wireless capsule endoscopy into image frames. A fuzzy logic technique and statistical features have been used to collect the features from the captured images. These statistical features are used in a QSVM classifier to detect bleeding and non-bleeding images from the wireless capsule endoscopy images.

\section{RESEARCH METHODOLOGY}

An exemplary method is proposed to detect the availability of blood portions in the image cells that are received from the wireless capsule endoscopy video. In this research work, a fuzzy logic edge detection technique has been used for identifying the corner edge of the abnormalities of the images so that only the abnormal section can be calculated. In this circumstance, bleeding features are considered as the abnormalities or the main informative portion to be extracted. The proposed algorithm has a simple framework to compute, which is very easy to calculate and time-saving as well. The complete layout of the proposed method is depicted in Figure 1. 


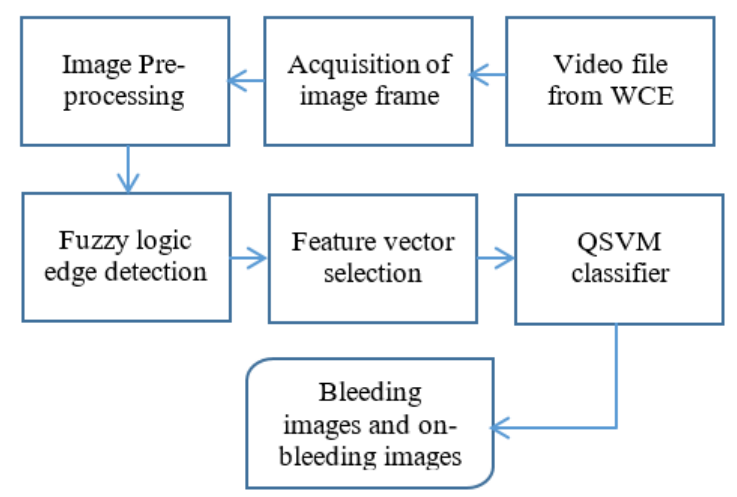

Figure 1. Schematic diagram of the proposed method

\subsection{Acquisition of image frame}

The actual data from the WCE of the GI tract is in the video form. Initially, a robust database of images folder has been created by converting the video clip obtained from the WCE into the image frame, which depends on the frame rate specified by WCE. The CE. PillCam*SB has been used as CE to take the GI tract video, whose longevity was 8 hours, and the frame rate was 2 , which is adequate to supply an enrich database for analyzing [2]. From the database, the annotated bleeding and non-bleeding dataset have been created for classifying the bleeding images from the non-bleeding image. Around 2393 annotated images have been used to analyze the proposed method, which is available in [23].

\subsection{Image pre-processing}

RGB is a compound color model consisting of three-color channels red, green, and blue. The captured image by WCE cameras is in RGB format. At first, we cut off around 33\% of the image so that we can remove the partial blackish portion and WCE tag from the image frame. After that, this RGB image is converted into a grayscale image so that we can take action on the two-dimensional array instead of a threedimensional array.

Furthermore, it is converted into a double-precision framework as a fuzzy logic speculator only in a double-precision structure. The output images have been adjusted in a range of $1 \%$ to make the intensity label between low and high. By the technique of unsharp masking, the output image has been tried to make sharper. The sequential process of image pre-processing is shown in Figure 2.

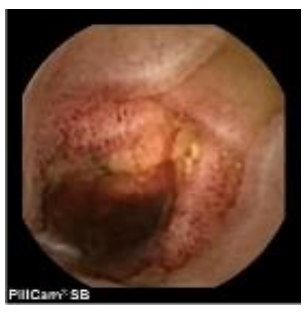

(a)

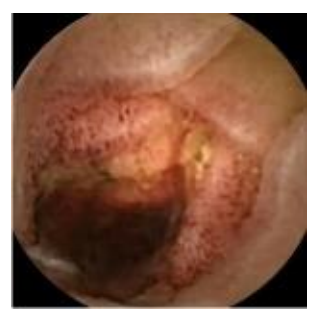

(b)

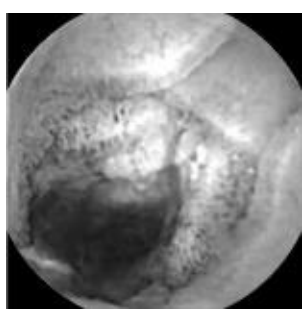

(c)

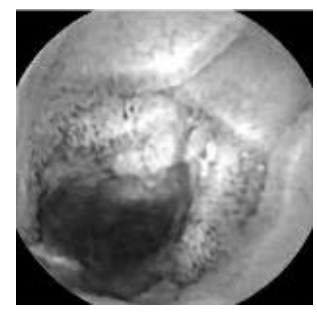

(d)

Figure 2. The sequential technique of image pre-processing; (a) primary sample bleeding image, (b) $33 \%$ cut of image, (c) adjusted image, (d) sharpen-image

\subsection{Fuzzy logic edge detection}

At the initial stage of the method, we have calculated the gradient of the image on the axis of $\mathrm{x}$ and $\mathrm{y}$ at where images have diversified from the identical region. Deviation from the uniform region indicates the variation of pixel intensity and area. Therefore, we have applied two gradient filters of the same range of $\left[\begin{array}{ll}-1 & 1\end{array}\right]$ and convolute with the images to find the gradient along the two axes. This convolution will return a subset following a structure like the center section of the convolution with the same size as the primary variable. Figure 3 shows the convolutional result along the axes. 
We have employed an edge detection fuzzy interface system in which it returns the fuzzy interface of Mamdani with indicated name. Then, we added the variables into the interface system by including convolutional results along the axes with specified boundary limits. After that, we have created membership function as zero (null) average Gaussian algorithm for individual inputs. It is characterized as zero membership type with degree 1 when the gradient value holds 0 for a pixel. Then, we summed this particular membership function into the interface system by including convolutional results along the axes with a specified value to be added to the membership function.

Besides, we have included zero and Gaussian membership functions with standard deviation for convolutional results along the axes for null membership function, which varies the intensity of the recognized edge. After that, we fixed [ $\left[\begin{array}{ll}0 & 1\end{array}\right]$ of the intensity of the edge perceived image from the immediate last action as output and also a member function of a triangle consisting of white and black values for output. These triangular white and black values $\left(w_{a}=1, w_{b}=1, w_{c}=2, b_{a}=0, b_{b}=0\right.$ and $b_{c}=0.3$ have a significant effect on the intensity of the recognized image. Last of all, a fuzzy interface system rules have been created as mentioned white for the identical region and black for the distinct region. Final edge recognized images were extracted for every row for the pixel in double type grey image evaluating prospective convolutional results along the axes. The above-specified Figure 4 shows the final extracted edge image from the sample data.

The output image has been further reconstructed to the RGB color image. Besides, the output image has been converted to an HSV color image for extracting the features from the HSV color space. The HSV color model has three color three channels of Hue, Saturation and value, which has been converted from the corresponding three RGB channels.
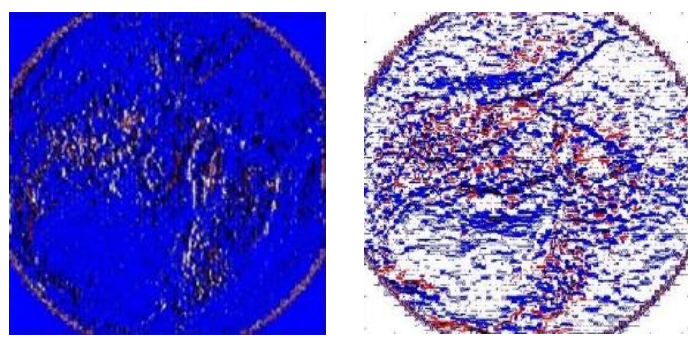

Figure 3. Convolutional values along (left) $\mathrm{x}$-axis and (right) $\mathrm{y}$-axis
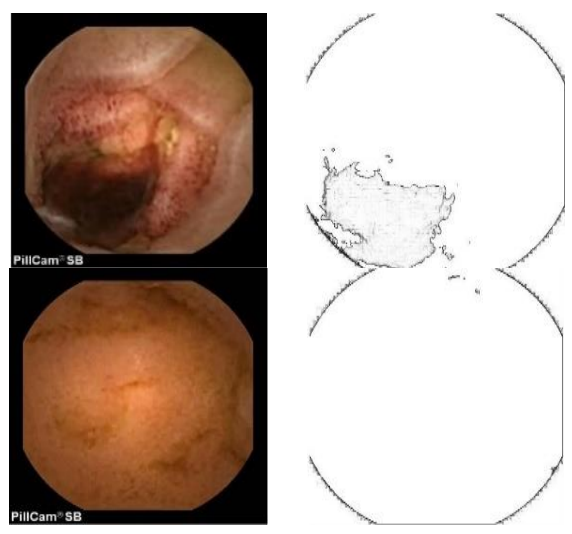

Figure 4. Edge recognized by the fuzzy logic system (Left top) and (Left bottom) represents sample images and (Right top) and (Right bottom) represents detected edge image for bleeding and non-bleeding image respectfully

\subsection{Feature selection}

As the RGB color space is a combination of three color channels, it can be described with every single color channel. The intensity of every color image has intensity between 0 and 255 of RGB components. The process of feature extraction can be referred to as the dimensional retrenchment that can be represented as a particular section of images efficiently in a specified vector of features. The proposed method used distinct statistical features like mean, mode, variance, entropy, skewness maxima, and moment 
to extract the features and make proper calculations from the segmented image. These characterized features are extracted from the proposed HSV color model as the HSV color model gives better possible results on the extracted features. The HSV color model can be obtained by converting the values of the corresponding three channels of the RGB color model [24].

Some statistical featured have been obtained from the above-segmented images for classifying the bleeding images from non-bleeding images. The features have been examined consecutively by principal component analysis (PCA) to find out the appropriate features from the images. PCA will incorporate the most correlated statistical features from the immense pool of feature vector. It will reduce the dimension of the vector by taking the most relevant combination of elements from the feature vector. Besides, it will also reduce the possibility of over fitting in the case of detecting the images. The statistical features that have been used in this proposed method are mean, mode, standard variation, skewness, entropy, variance, maxima, and moment.

\subsection{QSVM classifier}

Support vector machine is considered as one of the most common machine learning classifier techniques which belong to nonparametric arrangement for the sake of employing the function of the kernel. Vapnik was the pioneer who first proposed this classifier [25]. In the proposed method, the quadratic support vector machine technique has been used for differentiating the bleeding and non-bleeding objects. Say, we have considered $\mathrm{n}$ training data $\mathrm{b}_{\mathrm{i}}, b_{i} \in R^{N}$ for $i=1, \ldots n$ each either in ulcer or non-ulcer, then as separating rule.

$$
\omega^{T} x \geq \gamma
$$

where, $x \in \mathbb{R}^{N}$ is a variable with $\omega \in \mathbb{R}^{N}, \gamma \in \mathbb{R}$ is the classifier parameters. In the proposed technique, a kernel function has been used which is denoted by $K\left(x_{b l}, x_{n b l}\right)$ where $x_{b l}$ and $x_{n b l}$ mean bleeding and nonbleeding, respectively.

$$
K\left(x_{b l}, x_{n b l}\right)=\left(x_{b l} \cdot x_{n b l}\right)^{s}
$$

Where $S$ is a degree of the corresponding polynomial. And, the values of $\omega$ and $\gamma$ can be calculated from the equation (1),

$$
\min _{\omega \in \mathbb{R}^{N}, \gamma \in \mathbb{R}, y \in \mathbb{R}_{+}^{n}} \frac{1}{2} y^{T} D\left[K\left(x_{b l}, x_{n u l}\right) K\left(x_{b l}, x_{n u l}\right)\right] D y-C e^{T} y
$$

Where, $\omega=y^{T} D\left[K\left(x_{b l}, x_{n b l}\right) K\left(x_{b l}, x_{n b l}\right)\right] D y$

$$
\gamma=-e^{T} D y
$$

A validation technique is employed to find out the error rate of the proposed method, which is considered to be closed to the actual error of the method. 10-fold cross-validation has been examined to differentiate the samples into training and testing sets. The training and testing image ratios are $90 \%$ and $10 \%$, respectfully considering the 10 -fold cross-validation.

The output error obtained from this technique is the mean error rate of iterations. Holdout validation has been utilized in QSVM classifier in which the validation is performed repeatedly to get the optimal result. By employing the lamination technique, the false rate can be made more efficient [25]. The bleeding and non-bleeding images have successfully separated by implementing the Quadratic support vector machine.

\section{RESULTS AND DISCUSSION}

The performance of the diagnosis process of bleeding detection depends on a few attributes. The significant possible circumstances that may be happened during detecting bleeding images like True bleeding detection (TP), True non-bleeding detection (TN), false bleeding detection (FP), and false non-bleeding detection (FN). Since accuracy cannot be considered a reliable performance perimeter to evaluate research performance, some other performance matrices are used. We experimented with specificity, sensitivity, precision and negative predicted value F1score, to evaluate the research work's performance.

The proposed method achieved the best possible result of sensitivity 98\%, accuracy $98.2 \%$, specificity $98 \%$, negative predicted value $99 \%$, precision $93 \%$, and F1 Score $95.4 \%$ by utilizing the QSVM classifier with used 10-fold cross-validation technique which is depicted in Figure 5. Besides, a suitable combination of different statistical features has been generated by using principal component analysis (PCA). 
The comparison between different color models by implementing the QSVM classifier is showed in Table 1. From Table 1, it can be easily concluded that the proposed HSV color space is more superior to all other color spaces. All the performance parameters mentioned in Table 1 are better for the HSV color model than the different color models. Besides, Table 2 shows the performance comparison between distinct classifiers used to detect bleeding and non-bleeding images. Again, Table 2 provides evidence that the proposed QSVM classifier has a better result than all other classifiers.

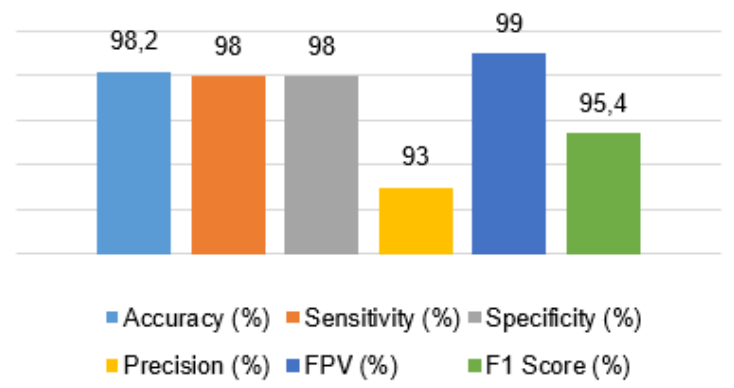

Figure 5. Performance result of the proposed method

Though revolutionary works have been implemented to detect the bleeding portion, the proposed method is unique and enriches the performance of this research field. A comparability study between the existing processes and the proposed method are presented in Table 3. Table 3 shows that the proposed technique is an improved edition of the bleeding detection research field in terms of accuracy and sensitivity compared with the existing work. Though Kundu et al. [26] examined 2300 color images from WCE for evaluating their bleeding detection algorithm, 2393 images have been studied in this proposed method, which is available in [23]. Also, this proposed method even analysis with some other additional performance parameters like precision, F1 Score and negative-predicted value. In a nutshell, the proposed method is an accurate model of bleeding detection.

Table 1. Performance comparison with distinct color models

\begin{tabular}{ccccccc}
\hline Color spaces & Accuracy & Sensitivity & Specificity & Precision & FPV & F1 Score \\
\hline HSV & $\mathbf{9 8 . 2}$ & $\mathbf{9 8}$ & $\mathbf{9 8}$ & $\mathbf{9 3}$ & $\mathbf{9 9}$ & $\mathbf{9 5 . 4}$ \\
RGB & 93.5 & 90 & 94 & 74 & 98 & 81.2 \\
L $^{*}$ *b $^{*}$ & 93 & 90 & 94 & 71 & 98 & 79.4 \\
YCbCr & 93.2 & 92 & 93 & 70 & 99 & 79.5 \\
\hline
\end{tabular}

Table 2. Performance comparison with distinct classifiers

\begin{tabular}{ccccccc}
\hline Different classifiers & Accuracy & Sensitivity & Specificity & Precision & FPV & F1 Score \\
\hline KNN & 98 & 96 & 97 & 91 & 99 & 93.4 \\
WKNN & 98.1 & 97 & 96 & 90 & 98 & 93.9 \\
QSVM (Proposed) & $\mathbf{9 8 . 2}$ & $\mathbf{9 8}$ & $\mathbf{9 8}$ & $\mathbf{9 3}$ & $\mathbf{9 9}$ & $\mathbf{9 5 . 4}$ \\
\hline
\end{tabular}

Table 3. Performance analysis with different existing techniques of bleeding detection

\begin{tabular}{cccc}
\hline Methodology & Accuracy & Sensitivity & Specificity \\
\hline Color information [10] & 97.7 & 96 & 91.3 \\
Uniform lbp [9] & 91.5 & 79.25 & 94.56 \\
Yiq stat [19] & 93.90 & 93.50 & 94.00 \\
R/g state [20] & 94.97 & 88.50 & 96.63 \\
Ratio of intensity [21] & 94.0 & 94.78 & 93.58 \\
Two-fold system [17] & 95.75 & 92 & 96.5 \\
Higher and lower order sate [2] & 97.96 & 97.75 & 97.99 \\
Proposed method & $\mathbf{9 8 . 2}$ & $\mathbf{9 8}$ & $\mathbf{9 8}$ \\
\hline
\end{tabular}

\section{CONCLUSION}

The proposed method provides a novel approach to detect the bleeding image from WCE images. The best possible result is obtained from the experiment using a Q-SVM classifier under the 10-fold crossvalidation, Fuzzy logic edge detection system with some processing, statistical feature vector using principal 
component analysis (PCA) in HSV color space. The proposed method has worked on 2393 WCE annotated images. The proposed method outcome with the highest sensitivity $98 \%$, accuracy $98.2 \%$, specificity $98 \%$, NPV 99\%, precision 93\%, and F1 Score 95.4\%. It can be concluded from this statistical result that the proposed method has higher efficiency to classify the bleeding and non-bleeding images than the existing comparable methods in terms of sensitivity, accuracy, F1 Score, and precision. Consequently, it has fulfilled the research objectives to deduce the physicians' burden by introducing an automated bleeding detection system. However, bleeding detection can be improved by examining different advanced feature learning and classifier techniques.

\section{ACKNOWLEDGEMENTS}

This research has been supported by Multimedia University (MMU) and the Malaysia Ministry of Higher 800 Education (Grant No: MMUE/180044).

\section{REFERENCES}

[1] A. L. M. Silveira, A. V. M. Ferreira, and M. M. Teixeira, "High-Fiber Diets in Gastrointestinal Tract Diseases," in Dietary Interventions in Gastrointestinal Diseases, pp. 229-244, 2019.

[2] T. Ghosh, S. A. Fattah, and K. A. Wahid, "Automatic Computer Aided Bleeding Detection Scheme for Wireless Capsule Endoscopy (WCE) Video Based on Higher and Lower Order Statistical Features in a Composite Color," Journal of Medical and Biological Engineering, vol. 38, no. 2, pp. 482-496, 2018.

[3] O. Olén, et al., "Increased Mortality of Patients with Childhood-Onset Inflammatory Bowel Diseases, Compared With the General Population," Gastroenterology, vol. 156, no. 3, pp. 614-622, 2019.

[4] G. G. Kaplan and S. C. Ng, "Understanding and Preventing the Global Increase of Inflammatory Bowel Disease," Gastroenterology, vol. 152, no. 2, pp. 313-321.e2, 2017.

[5] A. Wang, et al., "Wireless capsule endoscopy," Gastrointestinal Endoscopy, vol. 78, no. 6, pp. 805-815, 2013.

[6] G. Pan, G. Yan, X. Qiu, and J. Cui, "Bleeding detection in wireless capsule Endoscopy based on probabilistic neural network," Journal of Medical Systems, vol. 35, no. 6, pp. 1477-1484, 2011.

[7] J. M. Buscaglia, et al., "Performance Characteristics of the Suspected Blood Indicator Feature in Capsule Endoscopy According to Indication for Study," Clinical Gastroenterology and Hepatology, vol. 6, no. 3, pp. 298-301, 2008.

[8] S. Liangpunsakul, "Performance of given suspected blood indicator," The American Journal of Gastroenterology, vol. 98, no. 12, pp. 2676-2678, 2003.

[9] P. Sivakumar and B. M. Kumar, "A novel method to detect bleeding frame and region in wireless capsule endoscopy video," Cluster Computing, vol. 22, no. 5, pp. 12219-12225, 2019.

[10] K. Pogorelov, et al., "Bleeding detection in wireless capsule endoscopy videos-Color versus texture features," Journal of Applied Clinical Medical Physics, vol. 20, no. 8, pp. 141-154, 2019.

[11] A. R. Hassan, and M. A. Haque, "Computer-aided gastrointestinal hemorrhage detection in wireless capsule endoscopy videos," Computer Methods and Programs in Biomedicine, vol. 122, no. 3, pp. 341-353, 2015.

[12] Y. Yuan, B. Li, and M. Q. H. Meng, "Bleeding Frame and Region Detection in the Wireless Capsule Endoscopy Video," IEEE Journal of Biomedical and Health Informatics, vol. 20, no. 2, pp. 624-630, 2016.

[13] T. Ghosh, S. K. Bashar, S. A. Fattah, C. Shahnaz, and K. A. Wahid, "An automatic bleeding detection scheme in wireless capsule endoscopy based on statistical features in hue space," 2014 17th International Conference on Computer and Information Technology (ICCIT), Dhaka, 2014, pp. 354-357.

[14] T. Ghosh, et al., "An automatic bleeding detection technique in wireless capsule endoscopy from region of interest," 2015 IEEE International Conference on Digital Signal Processing (DSP), Singapore, 2015, pp. 1293-1297.

[15] T. Ghosh, et al., "A statistical feature based novel method to detect bleeding in wireless capsule endoscopy images," 2014 International Conference on Informatics, Electronics \& Vision (ICIEV), Dhaka, 2014, pp. 1-4.

[16] T. Ghosh, S. A. Fattah, C. Shahnaz, and K. A. Wahid, "An automatic bleeding detection scheme in wireless capsule endoscopy based on histogram of an RGB-indexed image," 2014 36th Annual International Conference of the IEEE Engineering in Medicine and Biology Society, Chicago, IL, 2014, pp. 4683-4686.

[17] A. Al Mamun and M. S. Hossain, "Ulcer Detection in Image Converted from Video Footage of Wireless Capsule Endoscopy," in 2019 1st International Conference on Advances in Science, Engineering and Robotics Technology (ICASERT), Dhaka, Bangladesh, 2019, pp. 1-4.

[18] M. S. Hossain, A. Al Mamun, M. G. Hasan, and M. M. Hossain, "Easy Scheme for Ulcer Detection in Wireless Capsule Endoscopy Images," 2019 1st International Conference on Advances in Science, Engineering and Robotics Technology (ICASERT), Dhaka, Bangladesh, 2019, pp. 1-5.

[19] A. Al Mamun, M. S. Hossain, M. M. Hossain, and M. G. Hasan, "Discretion Way for Bleeding Detection in Wireless Capsule Endoscopy Images," 2019 1st International Conference on Advances in Science, Engineering and Robotics Technology (ICASERT), Dhaka, Bangladesh, 2019, pp. 1-6.

[20] S. Narjim, A. Al Mamun, and D. Kundu, "Diagnosis of acute lymphoblastic leukemia from microscopic image of peripheral blood smear using image processing technique," ICONCS 2020: Cyber Security and Computer Science, vol. 325, pp. 515-526, 2020.

[21] M. S. Hossain, et al., "Ulcer detection in wireless capsule endoscopy using locally computed features," ICONCS 2020: Cyber Security and Computer Science, vol. 325, pp. 491-502, 2020. 
[22] A. Al Mamun, M. S. Hossain, M. E. Khallil, A. Tahabilder, T. K. Das, and R. Islam, "Convenient Way to Detect Ulcer in Wireless Capsule Endoscopy through Fuzzy Logic Technique," in 2020 IEEE Region 10 Symposium, TENSYMP 2020, Jun. 2020, pp. 880-883, doi: 10.1109/TENSYMP50017.2020.9231004.

[23] V. Charisis, L. Hadjileontiadis, and G. Sergiadis, "Enhanced Ulcer Recognition from Capsule Endoscopic Images Using Texture Analysis," in New Advances in the Basic and Clinical Gastroenterology, InTech, pp. 185-210, 2012.

[24] P. Białoń, "Solving Support Vector Machine with Many Examples," Journal of Telecommunications and Information Technology, pp. 65-70, 2010.

[25] A. Kumar, "Machine Learning: Validation Techniques," DZone AI, 2018.

[26] A. K. Kundu, S. A. Fattah, and M. N. Rizve, "An automatic bleeding frame and region detection scheme for wireless capsule endoscopy videos based on interplane intensity variation profile in normalized RGB color space," Journal of Healthcare Engineering, vol. 2018, 2018.

\section{BIOGRAPHIES OF AUTHORS}
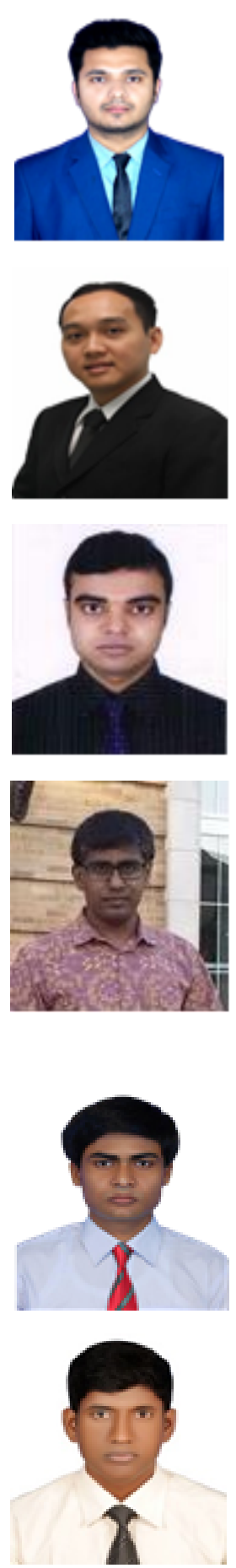

Abdullah Al Mamun has received a B.Sc. degree in Electrical and Electronic Engineering from Pabna University of Science and Technology in 2018. Now he is pursuing M.Eng.Sc.at Multimedia University (MMU) in the Faculty of Engineering and Technology since 2019. He is also working as a lecturer (on study leave) since November 2019 in EEE at Feni University, Feni, Bangladesh. His research interest includes computer vision; image processing, signal processing, deep learning and machine learning.

Em Poh Ping is currently a lecturer at the Faculty of Engineering and Technology, Multimedia University. Poh Ping researches vehicle safety, data fusion, fuzzy control, and image processing. His current project is "lane departure estimation using vision and vehicle dynamical's state."

Tonmoy Ghosh received the B.Sc. and M.Sc. degree in Electrical and Electronic Engineering (EEE) from the Bangladesh University of Engineering and Technology (BUET), Dhaka, Bangladesh, in 2012 and 2016, respectively. He joined as a Lecturer in the EEE department at Pabna University of Science and Technology (PUST), Pabna, Bangladesh. Currently, He is doing his $\mathrm{PhD}$ in the department of ECE at The University of Alabama, Tuscaloosa, USA. His research interests include image processing, pattern recognition, and digital signal processing.

Md Motaher Hossain received the bachelor's degree in electrical and electronic engineering from the Rajshahi University of Engineering and Technology (RUET), Rajshahi, Bangladesh, in 2007, the M.Sc. degree in Renewable Energy from the Carl von Ossietzky Universität Oldenburg, Oldenburg, Germany in 2010. He is currently perusing another M.Sc. degree from the Engineering Technologies Department at Bowling Green State University, OH, USA. He has been working as an Assistant Professor (on study leave) in the Department of Electrical and Electronic Engineering at Pabna University of Science and Technology, Pabna, Bangladesh since 11 May 2013. His research interests include Power Systems, Power Electronics, Renewable Energy, Control System Engineering, and Deep Learning.

Md. Galib Hasan has been graduated from Khulna University of Engineering and Technology. His major is Electrical and Electronic Engineering (EEE) and currently he is working as an assistant professor in the department of EEE at Pabna University of Science and Technology. His research interest includes digital image processing, embedded systems, VLSI, Chip

Md. Golam Sadeque has received B. Sc. Eng. degree in Electrical and Electronics Engineering (EEE) from Rajshahi University of Engineering and Technology (RUET) in 2010. He is pursuing a master's of engineering science (M. Eng. Sc,) degree under the faculty of engineering at Multimedia University. He is also working as an Assistant professor (on study leave) since 23 June 2015 in the department of EEE at Pabna University of Science and Technology (PUST), Pabna-6600, Bangladesh. His research interest includes the design of Radiofrequency power amplifier (RFPA) and Biomedical engineering. E-mail: golamsadeq@gmail.com. 